\title{
Linear precoder design for non-orthogonal AF MIMO relaying systems based on MMSE criterion
}

\author{
Yunida Yunida, Nasaruddin Nasaruddin * (D), Rusdha Muharar and Yuwaldi Away
}

\begin{abstract}
Multiple-input multiple-output (MIMO) relaying system has attracted the attention of cooperative network researchers, due to its advantage over the conventional single antenna system, in terms of system capacity and spatial diversity. Precoder design is a processing scheme implemented at a source and relay node to improve system performance. We propose a linear precoder design for non-orthogonal amplify-and-forward MIMO relaying systems based on the minimum mean square error (MMSE) criterion. We analyze an upper bound of MMSE using a convenient expression to determine the structure of precoding matrices using the singular-value decomposition technique. Simulation results demonstrate that the proposed precoded scheme outperforms both unprecoded and existing precoded schemes.
\end{abstract}

Keywords: MIMO relaying, Cooperative network, Precoder design, Non-orthogonal amplify-and-forward, MMSE criterion

\section{Introduction}

Relaying techniques in cooperative wireless networks increase a network's coverage area and data rate and reduce transmission power $[1,2]$. Generally, improving the performance of cooperative networks can be achieved by relaying protocols such as the decode-and-forward (DF) [3, 4] and amplify-and-forward (AF) [5-7] schemes. The DF scheme is complex because the relay node must first decode the signal received from a source and then transmit the re-encoded signal to the destination. In contrast, the AF scheme is simple to implement because the relay node only needs to transmit an amplified version of the received signal. Among different AF schemes, half-duplex non-orthogonal AF (NAF) relaying [8, 9] has been considered a superior scheme. With NAF relaying, received collision and the broadcast range can be maximized because the relay and source nodes can transmit signals simultaneously in both cooperative and broadcasting phases.

Furthermore, the capacity of cooperative networks can be increased using multiple antennas at the transceiver, i.e., multiple-input multiple-output (MIMO) relaying techniques [10, 11]. Recently, seminal studies into MIMO relaying systems have concentrated on a transceiver

\footnotetext{
* Correspondence: nasaruddin@unsyiah.ac.id

Electrical and Computer Engineering Department, Syiah Kuala University, Banda Aceh 23111, Indonesia
}

precoding design with orthogonal or conventional AF schemes [12-14]. A transceiver precoding design is a processing technique that exploits channel state information (CSI) by weighting information streams at the transmitter to achieve transmit diversity. Mo and Chew [12] proposed two schemes of precoding design for AF MIMO relay networks under minimum mean square error (MMSE) criterion and QoS requirements, i.e., optimal joint source and relay precoding (OJSRP) and suboptimal relay only precoding (SROP) schemes. They proved that the OJSRP scheme outperforms the SROP scheme in terms of MSE performance and capacity, which shows that precoding at both source and relay nodes achieves an improved (higher) performance compared to precoding only at the relay nodes. To optimize the power distribution between the source and relay nodes in the OJSRP scheme, a joint precoder design and node power allocation based on the MSE criterion in AF MIMO relay networks has been considered [13]. This approach demonstrated that the most efficient method to allocate node power is adjusting the noise level of the receiver nodes. A detailed investigation of the diverse linear precoder designs for AF MIMO relay networks based on MMSE, zero-forcing (ZF), and maximum information rate (MIR) criteria can be found in the literature [14]. In that study, the results showed that the MMSE criterion achieves near-optimal performance 
advantage compared to the ZF and MIR criteria when channel coding with a low coding rate is applied jointly across the source antennas.

Motivated by the above advantages of the MMSE criterion in AF MIMO networks, we propose a linear precoder design for half-duplex NAF MIMO relaying networks based on the MMSE criterion. To the best our knowledge, such a precoder design for non-orthogonal AF MIMO relay schemes has not been proposed. Some studies $[15,16]$ have investigated precoder designs for three-node NAF relaying systems where all nodes are equipped with a single antenna. The optimal precoder detectors based on MMSE and minimum bit error rate (MBER) criteria have also been proposed in [15]. That study concluded that the proposed detectors outperformed other optimal detectors, i.e., channel inversion, maximum ratio combining, and biased maximum likelihood detectors. Compared to an MBER detector [15], the MMSE detector cannot achieve full diversity; however, the computational complexity of the MMSE detector is stable relative to the constellation size of the modulation schemes, while the MBER detector increases significantly [16] in terms of bit error rate (BER) performance. Our main contributions and solutions in this paper can be summarized as:

- A precoder design for non-orthogonal AF MIMO relay schemes using the MMSE criterion

- A simpler suboptimal solution to the optimization problem of the precoder design by optimizing the transmit power from source and destination

- An analysis of the system performance of the proposed scheme for unprecoded and precoded NAF MIMO relaying systems.

The remainder of this paper is organized as follows. A system model and the proposed precoder design with NAF relaying are introduced in Sections 2 and 3, respectively. Simulation results are provided in Section 4, and a brief conclusion is presented in Section 5 .
Notations: In this paper, matrices and vectors are in boldface. ( $)^{H}$ and $\mathbf{I}_{N}$ denote the Hermitian transpose of a vector and identity the matrix of size $N$, respectively, while $E($.$) and \operatorname{tr}($.) denote the expectation and trace functions, respectively.

\section{Proposed system model and problem formulation \\ 2.1 Precoder for NAF MIMO relaying system}

A three-node MIMO non-orthogonal relaying system comprised a source $(S)$, a relay node $(R)$, and a destination $(D)$ shown in Fig. 1. This system is equipped with multiple antennas, i.e., $N_{S}, N_{R}$, and $N_{D}$, that cannot transmit or receive signals simultaneously. In other words, each node communicates in half-duplex mode. The transmission process of a non-orthogonal relaying system is divided into broadcasting and cooperative phases.

In the broadcasting phase, $S$ precodes the signal vector $s_{1} \in \mathcal{E}^{N_{B} \times 1}$ linearly using a precoding matrix $\mathbf{F}_{S} \in \mathcal{E}^{N_{S} \times N_{B}}$ where $N_{B} \leq \min \left(N_{S}, N_{R}, N_{D}\right)$ is the number of data streams. Then, $S$ transmits it to $R$ and to $D$ directly. The broadcast signal vector received by both $D$ and $R$ can be formulated respectively as follows:

$$
\mathbf{y}_{D, 1}=\mathbf{H}_{S D} \mathbf{F}_{S^{s_{1}}}+\mathbf{n}_{D, 1}
$$

and

$$
\mathbf{y}_{R}=\mathbf{H}_{S R} \mathbf{F}_{S^{s_{1}}}+\mathbf{n}_{R}
$$

Here, $\mathbf{y}_{D, 1} \in \mathbb{C}^{N_{D} \times 1}$ and $\mathbf{y}_{R} \in \mathbb{C}^{N_{R} \times 1}$ are the received signal vectors at $D$ and $R$, respectively, while $\mathbf{H}_{S D} \in \mathbb{C}^{N_{D} \times N_{S}}$ and $\mathbf{H}_{S R} \in \mathbb{C}^{N_{R} \times N_{S}}$ are the complex Gaussian random MIMO channel gain matrices of the $S \rightarrow D$ and $S \rightarrow R$ links, respectively. The noise vector $\mathbf{n}_{D, 1} \in \mathbb{C}^{N_{D} \times 1}$ and $\mathbf{n}_{R} \in \mathbb{C}^{N_{R} \times 1}$ are the respective additive white Gaussian noise (AWGN) samples at the receiving antennas of $D$ and $R$, respectively, which are assumed to have independent identically distributed (i.i.d) components with zero-mean and covariance matrices $E\left[\mathbf{n}_{D, 1} \mathbf{n}_{D, 1}^{H}\right]=\sigma_{n, d}^{2} \mathbf{I}_{N_{D}}$

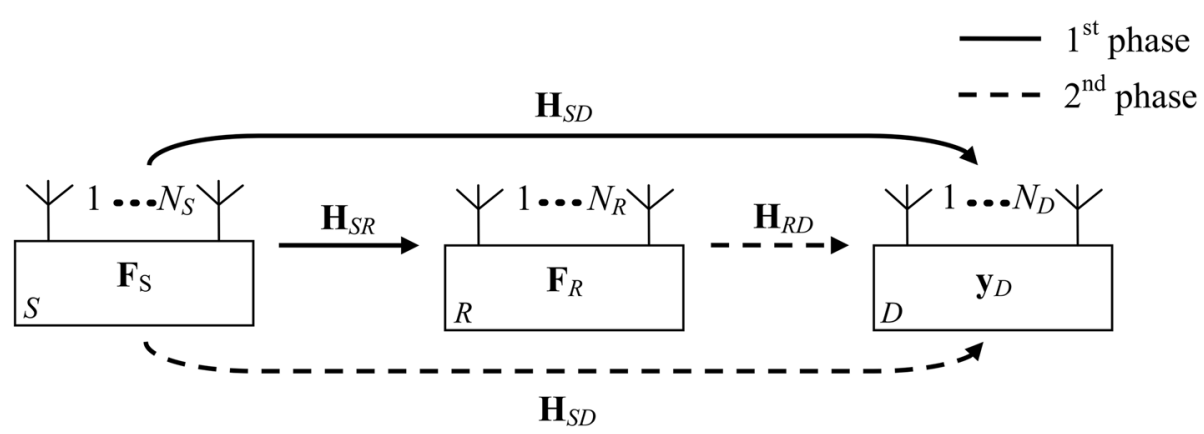

Fig. 1 Half-duplex non-orthogonal MIMO relaying system 
and $E\left[\mathbf{n}_{R} \mathbf{n}_{R}^{H}\right]=\sigma_{n, r}^{2} \mathbf{I}_{N_{R}}$, where $\sigma_{n, d}^{2}$ and $\sigma_{n, r}^{2}$ are the noise variances at $D$ and $R$, respectively.

In the cooperative phase, $R$ linearly multiplies the received signal in (2) by a precoding matrix $F_{R} \in \mathcal{E}^{N_{R} \times N_{R}}$ and transmits this amplified signal vector to $D$. By assuming a non-orthogonal cooperative system, $R$ communicates with the destination and $S$ transmits the signal vector $\mathbf{s}_{2} \in \mathcal{E}^{N_{B} \times 1}$ to $D$. Thus, $D$ receives the signal vectors from $S$ and $D$ as follows:

$$
\begin{aligned}
\mathbf{y}_{D, 2} & =\mathbf{H}_{R D} \mathbf{F}_{R} \mathbf{y}_{R}+\mathbf{H}_{S D} \mathbf{F}_{S^{s_{2}}}+\mathbf{n}_{D, 2} \\
& =\mathbf{H}_{R D} \mathbf{F}_{R} \mathbf{H}_{S R} \mathbf{F}_{S^{s_{1}}}+\mathbf{H}_{S D} \mathbf{F}_{s^{s_{2}}}+\left(\mathbf{H}_{R D} \mathbf{F}_{R} \mathbf{n}_{R}+\mathbf{n}_{D, 2}\right),
\end{aligned}
$$

where $\mathbf{H}_{R D} \in \mathcal{E}^{N_{D} \times N_{R}}$ is the complex Gaussian random MIMO channel gain matrix of the $R \rightarrow D$ link. The noise vector $\mathbf{n}_{D, 2} \in \mathcal{E}^{N_{D} \times 1}$ is the AWGN samples at the receiving antennas of $D$ with zero-mean and covariance matrix $E\left[\mathbf{n}_{D, 2} \mathbf{n}_{D, 2}^{H}\right]=\sigma_{n, d}^{2} \mathbf{I}_{N_{D}}$. The two received signal vectors at $D$, i.e., $\mathbf{y}_{D, 1}$ and $\mathbf{y}_{D, 2}$, can be combined into a single vector denoted as $\mathbf{y}_{D} \in E^{2 N_{D} \times 1}$. Thus, (3) and (1) can be rewritten as follows:

$$
\mathbf{y}_{D}=\left[\begin{array}{l}
\mathbf{y}_{D, 1} \\
\mathbf{y}_{D, 2}
\end{array}\right]=\mathbf{H F}_{S} \mathbf{s}+\mathbf{n}
$$

where

$$
\mathbf{H}=\left[\begin{array}{cc}
\mathbf{H}_{S D} & \mathbf{0} \\
\mathbf{H}_{R D} \mathbf{F}_{R} \mathbf{H}_{S R} & \mathbf{H}_{S D}
\end{array}\right], \mathbf{s}=\left[\begin{array}{l}
\mathbf{s}_{1} \\
\mathbf{s}_{2}
\end{array}\right] \text { and } \mathbf{n}=\left[\begin{array}{c}
\mathbf{n}_{D, 1} \\
\mathbf{H}_{R D} \mathbf{F}_{R} \mathbf{n}_{R}+\mathbf{n}_{D, 2}
\end{array}\right] .
$$

\subsection{MMSE receiver and related MSE matrix}

In this subsection, we experimentally investigate a linear precoder design in terms of the MMSE criteria. Here, we consider that a weighting matrix, $\mathbf{W} \in £^{N_{D} \times N_{B}}$, is used to detect the received data stream at $D$.

$$
\hat{\mathbf{s}}=\mathbf{W}^{H} \mathbf{y}_{D}
$$

Under the assumption that CSI is available at all nodes, $\mathbf{F}_{S}, \mathbf{F}_{R}$, and $\mathbf{W}$ can be adjusted to each channel recognition to increase the performance of the NAF MIMO relaying systems. The MSE matrix, which indicates the covariance matrix of the symbol detection error of the data streams, is expressed as follows:

$$
M=E\left\{\|\hat{s}-s\|^{2}\right\}
$$

The transmitted symbol $\mathbf{s}$ is assumed to be i.i.d with zero-mean and covariance matrix $\mathbf{R}_{s s}=E\left[\mathbf{s s}^{H}\right]=2 \sigma_{s}^{2} \mathbf{I}_{N_{B}}$, with $E\left[\mathbf{s}_{1} \mathbf{s}_{1}^{H}\right]=E\left[\mathbf{s}_{2} \mathbf{s}_{2}^{H}\right]=\sigma_{s}^{2} \mathbf{I}_{N_{B}}$, where $\sigma_{s}^{2}$ is the power of the transmitted symbols. The covariance matrix of the equivalent noise vector in (4) can be written as follows:
$\mathbf{R}_{n n}=E\left[\mathbf{n n}^{H}\right]=\left[\begin{array}{cc}\sigma_{n, d}^{2} \mathbf{I}_{N_{D}} & 0 \\ 0 & \sigma_{n, r}^{2} \mathbf{H}_{R D} \mathbf{F}_{R} \mathbf{F}_{R}^{H} \mathbf{H}_{R D}^{H}+\sigma_{n, d}^{2} \mathbf{I}_{N_{D}}\end{array}\right]$.

By substituting (5) into (6) and starting with computing the squared error, the optimal estimator can be expressed as follows:

$$
\mathbf{W}=2 \sigma_{s}^{2}\left(2 \sigma_{s}^{2} \mathbf{H F}_{S} \mathbf{F}_{S}^{H} \mathbf{H}^{H}+\mathbf{R}_{n n}\right)^{-1} \mathbf{H F}_{S}
$$

where $\mathbf{E}\left[\mathbf{y}_{D} \mathbf{y}_{D}^{H}\right]=2 \sigma_{s}^{2} \mathbf{H F}_{S} \mathbf{F}_{S}^{H} \mathbf{H}^{H}+\mathbf{R}_{n n}$ is the covariance matrix of the received symbol vector at $D$. Given $\mathbf{W}$ in (8), the MSE of the estimate symbols in (6) can be written as:

$$
\hat{\mathbf{s}}=2 \sigma_{s}^{2} \mathbf{H}^{H} \mathbf{F}_{S}^{H}\left(2 \sigma_{s}^{2} \mathbf{H} \mathbf{F}_{S} \mathbf{F}_{S}^{H} \mathbf{H}^{H}+\mathbf{R}_{n n}\right)^{-1} \mathbf{y}_{D}
$$

By substituting (9) into (6), and minimizing and performing the matrix inversion lemma [17], the MMSE is formulated as follows:

$$
M_{\min }=\operatorname{tr}\{\mathbf{A}\}=\operatorname{tr}\left\{\left(2 \sigma_{s}^{-2} \mathbf{I}_{N_{B}}+\mathbf{A}_{R}+\mathbf{A}_{S}\right)^{-1}\right\},
$$

where

$$
\begin{aligned}
\mathbf{A}_{R}= & \mathbf{F}_{S}^{H} \mathbf{H}_{S R}^{H} \mathbf{F}_{R}^{H} \mathbf{H}_{R D}^{H}\left(\sigma_{n, r}^{2} \mathbf{H}_{R D} \mathbf{F}_{R} \mathbf{F}_{R}^{H} \mathbf{H}_{R D}^{H}+\sigma_{n, d}^{2} \mathbf{I}_{N_{D}}\right)^{-1} \\
& \times \mathbf{H}_{R D} \mathbf{F}_{R} \mathbf{H}_{S R} \mathbf{F}_{S}
\end{aligned}
$$

and

$$
\mathbf{A}_{S}=2 \sigma_{n, d}^{-2} \mathbf{F}_{S}^{H} \mathbf{H}_{S D}^{H} \mathbf{H}_{S D} \mathbf{F}_{S}
$$

The functions $A_{R}$ and $A_{S}$ account for the MMSE in the relay link and the direct link in both phases.

\subsection{Problem formulation}

As we can see in (10), the MMSE formula is the function of precoding matrices $F_{R}$ and $F_{S}$. Our next task is to design these two precoding matrices so that the MSE in (10) can be minimized. The optimization problem can be formulated as follows:

$$
\begin{array}{cc}
\min _{\mathbf{F}_{S}, \mathbf{F}_{R}} & \operatorname{tr}\{A\}=\sum_{i=1}^{N_{B}} A_{(i, i)} \\
\text { s.t. } & P_{S}+P_{R} \leq P_{\text {total }}
\end{array}
$$

where 


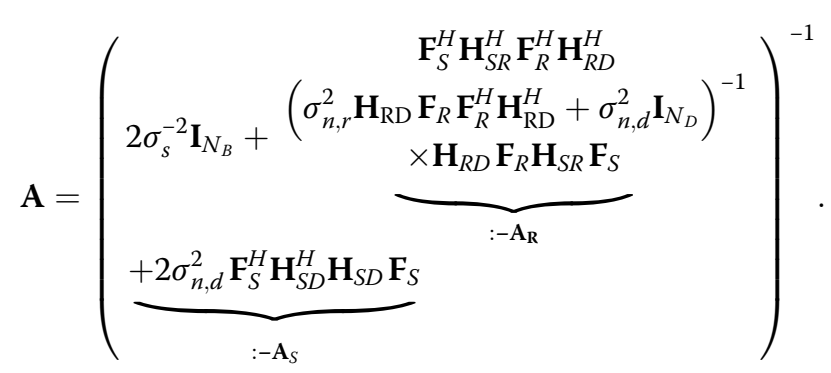

The function $P_{\text {total }}$ is the total transmission power at $S$ and $R$, while $P_{S}$ and $P_{R}$ are the respective transmit powers from $S$ and $R$ expressed as follows:

$$
\begin{aligned}
P_{S} & =\operatorname{tr}\left\{\mathbf{F}_{S} E\left[s s^{H}\right] \mathbf{F}_{S}^{H}\right\}=2 \sigma_{s}^{2} \operatorname{tr}\left\{\mathbf{F}_{S} \mathbf{F}_{S}^{H}\right\}, \\
P_{R} & =\operatorname{tr}\left\{E\left[\mathbf{F}_{R} y_{R} y_{R}^{H} \mathbf{F}_{R}^{H}\right]\right\} \\
& =\operatorname{tr}\left\{\mathbf{F}_{R}\left(\sigma_{n, r}^{2} \mathbf{I}_{N_{R}}+\sigma_{s}^{2} \mathbf{H}_{S R} \mathbf{F}_{S} \mathbf{F}_{S}^{H} \mathbf{H}_{S R}^{H}\right) \mathbf{F}_{R}^{H}\right\} .
\end{aligned}
$$

The inequalities in (13) show that the precoders have to fulfill the transmit power constraints at both the relay and the source node. From (13)-(16), we can immediately conclude that the solution is not a convex optimization. Moreover, the cost function implicates a series of matrix inversions and multiplications; it is a complex and nonlinear function of $\boldsymbol{F}_{S}$ and $\boldsymbol{F}_{R}$. We will propose a method, which is depicted below, to resolve these problems.

\section{Linear precoder design with MMSE criterion}

\subsection{Proposed approach}

The linear MMSE precoder can be achieved analytically using an MSE matrix diagonalization procedure [18]. Inspired by this procedure, in this section, we propose to carry out similar matrix diagonalization in our design. The trace operation in (13) can be performed easily if error matrix $\mathbf{A}$ can be diagonalized and the entire problem can be shorted. First, we consider singular-value decomposition (SVD) for the MIMO channel matrices in all links:

$$
\begin{aligned}
& \mathbf{H}_{S D}=\mathbf{U}_{s d} \sum_{s d} \mathbf{V}_{s d}^{H}, \\
& \mathbf{H}_{S R}=\mathbf{U}_{s r} \sum_{s r} \mathbf{V}_{s r}^{H}, \\
& \mathbf{H}_{R D}=\mathbf{U}_{r d} \sum_{r d} \mathbf{V}_{r d}^{H},
\end{aligned}
$$

where $\mathbf{U}_{s d} \in \mathcal{E}^{N_{D} \times N_{D}}, \mathbf{U}_{s r} \in \mathcal{E}^{N_{R} \times N_{R}}$, and $\mathbf{U}_{r d} \in \mathcal{E}^{N_{D} \times N_{D}}$ are the orthonormal left singular matrices of $\mathbf{H}_{S D}, \mathbf{H}_{S R}$, and $\mathbf{H}_{R D}$, respectively; $\sum_{s d} \in \mathrm{i}^{N_{D} \times N_{D}}, \sum_{s r} \in \mathrm{i}^{N_{R} \times N_{R}}$, and $\sum_{r d} \in \mathrm{i}^{N_{D} \times N_{D}}$ are the diagonal singular-value matrices of $\mathbf{H}_{S D}, \mathbf{H}_{S R}$, and $\mathbf{H}_{R D}$, respectively; and $\mathbf{V}_{s d} \in \mathcal{E}^{N_{S} \times N_{S}}, \mathbf{V}_{s r} \in$
$£^{N_{S} \times N_{S}}$, and $\mathbf{V}_{r d} \in E^{N_{R} \times N_{R}}$ are the unitary right singular matrices of $\mathbf{H}_{S D}, \mathbf{H}_{S R}$, and $\mathbf{H}_{R D}$, respectively.

By using the procedure described in the literature [18], we can determine the following:

$$
\begin{aligned}
& \mathbf{F}_{R}=\mathbf{V}_{r d} \sum_{r} \mathbf{U}_{r}=\mathbf{V}_{r d} \sum_{r} \mathbf{U}_{s r}^{H}, \\
& \mathbf{F}_{S}=\mathbf{V}_{s r} \sum_{S} \mathbf{U}_{S}
\end{aligned}
$$

After some calculations and manipulations by substituting (17)-(21) into (14), we obtain the MSE in (13) as follows:

$$
\begin{aligned}
\operatorname{tr}\{\mathbf{A}\}= & \operatorname{tr}\left\{\left(2 \sigma_{s}^{-2} \mathbf{I}_{N_{B}}+\boldsymbol{\Sigma}_{s}^{H} \boldsymbol{\Sigma}_{s r}^{H} \boldsymbol{\Sigma}_{r}^{H} \boldsymbol{\Sigma}_{r d}^{H}\left(\sigma_{n, r}^{2} \boldsymbol{\Sigma}_{r d} \boldsymbol{\Sigma}_{r} \boldsymbol{\Sigma}_{r}^{H} \boldsymbol{\Sigma}_{r d}^{H}\right.\right.\right. \\
& \left.+\sigma_{n, d}^{2} \mathbf{I}_{N_{D}}\right)^{-1} \boldsymbol{\Sigma}_{r d} \boldsymbol{\Sigma}_{r} \boldsymbol{\Sigma}_{s r} \boldsymbol{\Sigma}_{s} \\
& \left.\left.+2 \sigma_{n, d}^{-2} \boldsymbol{\Sigma}_{s}^{H} \mathbf{V}_{s d} \mathbf{V}_{s r}^{H} \boldsymbol{\Sigma}_{s d}^{H} \boldsymbol{\Sigma}_{s d} \mathbf{V}_{s d}^{H} \mathbf{V}_{s r} \boldsymbol{\Sigma}_{s}\right)^{-1}\right\} \\
= & \operatorname{tr}\left(\mathbf{P}^{-1}\right)-\operatorname{tr}\left(\mathbf{P}^{-1} \boldsymbol{\Sigma}_{s}^{H}\left(\mathbf{Q}^{-1}+\boldsymbol{\Sigma}_{s} \mathbf{P}^{-1} \boldsymbol{\Sigma}_{s}^{H}\right)^{-1} \boldsymbol{\Sigma}_{s} \mathbf{P}^{-1}\right),
\end{aligned}
$$

where

$$
\begin{aligned}
\mathbf{P} & =2 \sigma_{s}^{-2} \mathbf{I}_{N_{B}} \\
& +\boldsymbol{\Sigma}_{s}^{H} \boldsymbol{\Sigma}_{s r}^{H} \boldsymbol{\Sigma}_{r}^{H} \boldsymbol{\Sigma}_{r d}^{H}\left(\sigma_{n, r}^{2} \boldsymbol{\Sigma}_{r d} \boldsymbol{\Sigma}_{r} \boldsymbol{\Sigma}_{r}^{H} \boldsymbol{\Sigma}_{r d}^{H}+\sigma_{n, d}^{2} \mathbf{I}_{N_{D}}\right)^{-1} \boldsymbol{\Sigma}_{r d} \boldsymbol{\Sigma}_{r} \boldsymbol{\Sigma}_{s r} \boldsymbol{\Sigma}_{s}
\end{aligned}
$$

and

$$
\mathbf{Q}=2 \sigma_{n, d}^{-2} \sum_{S}^{H} \mathbf{V}_{s d} \mathbf{V}_{s r}^{H} \sum_{s d}^{H} \sum_{s d} \mathbf{V}_{s d}^{H} \mathbf{V}_{s r} \sum_{s}
$$

Using the matrix inverse lemma [18] to diagonalize matrix $\mathbf{Q}$, the MSE in (22) is as follows:

$$
\begin{aligned}
\operatorname{tr}(\mathbf{A}) & \leq \operatorname{tr}\left(\mathbf{P}^{-1}\right)-\operatorname{tr}\left(\mathbf{P}-1 \sum_{s}^{H}\left(Q^{-1}+\sum_{s} \mathbf{P}^{-1} \sum_{s}^{H}\right)^{-1} \sum_{s} \mathbf{P}^{-1}\right) \\
& =\sum_{i=1}^{N_{B}} \frac{1}{2 \sigma_{s}^{-2}+\frac{\sigma_{s, i}^{2} \sigma_{s r, i}^{2} \sigma_{r, i}^{2} \sigma_{r d, i}^{2}}{\sigma_{n, r}^{2} \sigma_{r d, i}^{2} \sigma_{r, i}^{2}+\sigma_{n, d}^{2}}+\sigma_{s, i}^{2}\left(\mathbf{Q}^{-1}(i, i)\right)^{-1}}
\end{aligned}
$$

where $\sigma_{s, i}^{2}$ and $\sigma_{r, i}^{2}$ are the transmit power of nodes $S$ and $R$, respectively. The result admits that the upper bound of the MSE function in (25) is a much simpler form than the original MSE in functions (13)-(14). Then, we achieve the precoder design by minimizing (25). Here, let $\sigma_{s, i}^{2}=p_{s, i}$ and $\sigma_{r, i}^{2}=p_{r, i}$, and the optimization of the MMSE criterion in (13) can be formulated as follows: 


$$
\begin{aligned}
& \min _{\substack{p_{s, i}, p_{r, i}, i=1, \ldots, N_{B}}} \sum_{i=1}^{N_{B}} \frac{1}{2 \sigma_{s}^{-2}+\frac{p_{s, i} \sigma_{s r, i}^{2} p_{r, i} \sigma_{r d, i}^{2}}{\sigma_{n, r}^{2} \sigma_{r d, i}^{2} p_{r, i}+\sigma_{n, d}^{2}}+p_{s, i}\left(\mathbf{Q}^{-1}(i, i)\right)^{-1}} \\
& \text { s.t } \underbrace{2 \sigma_{s}^{2} \sum_{i=1}^{N_{B}} p_{s, i}+\sum_{P_{B}}^{N_{B}} p_{r, i}\left(\sigma_{n, r}^{2}+\sigma_{s}^{2} \sigma_{s r, i}^{2} p_{s, i}\right) \leq P_{\text {total }}}_{P_{S}} \\
& p_{s, i} \geq 0, p_{r, i} \geq 0, \quad \forall_{i} \in\left\{1,2, \ldots, N_{B}\right\} .
\end{aligned}
$$

\subsection{Power optimization}

Furthermore, the optimization problem in (26) is a non-convex problem either, and its optimum solution is difficult to solve. Previously, the iterative water-filling technique was employed to find the optimal solution for the MMSE design [18]. In this paper, we propose a simpler suboptimal solution to the optimization problem of the precoder in (26). The proposed precoding method is determined by optimizing the transmit power from $S$ and $R$, i.e., $p_{s, i}$ and $p_{r, i}$, that can satisfy minimization of the upper bound of the MSE.

To minimize the MSE function in (26), we consider a constant function $\lambda$ for different indexes of $i$ :

$$
\lambda=2 \sigma_{s}^{-2}+\frac{p_{s, i} \sigma_{s r, i}^{2} p_{r, i} \sigma_{r d, i}^{2}}{\sigma_{n, r}^{2} \sigma_{r d, i}^{2} p_{r, i}+\sigma_{n, d}^{2}}+p_{s, i}\left(\mathbf{Q}^{-1}(i, i)\right)^{-1} .
$$

While maximizing $\lambda$, the optimal solution to (26) satisfies (27); therefore, we suggest the relay transmit power as follows:

$$
p_{r, i}=\frac{\Gamma}{\sigma_{s r, i}^{2}} \quad \forall_{i}=\left\{1,2, \ldots, N_{B}\right\},
$$

where $\Gamma$ is a coefficient set to satisfy the total transmission power $P_{\text {total }}$ in (26). This coefficient can be obtained by substituting (28) into (26) as follows:

$$
\Gamma=\frac{2\left(P_{\text {total }}-P_{S}\right)}{2 \sigma_{n, r}^{2} \sum_{i=1}^{N_{B}} \sigma_{s r, i}^{-2}+P_{S}} .
$$

By substituting (28) into (29), we obtain the source transmit power as follows:

$$
p_{s, i}=\frac{\lambda}{\frac{\Gamma \sigma_{r d, i}^{2}}{\sigma_{n, r}^{2} \Gamma \sigma_{s r, i}^{-2} \sigma_{r d, i}^{2}+\sigma_{n, d}^{2}}+\left(\mathbf{Q}^{-1}(i, i)\right)^{-1}} .
$$

The constant in (27) can be rewritten as a function of the total source transmit power by substituting $p_{s, i}$ in (30) into (26) as follows:

$$
\lambda=\frac{P_{S}}{2 \sigma_{s}^{2} \sum_{i=1}^{N_{B}}\left(\frac{\Gamma \sigma_{r d, i}^{2}}{\sigma_{n, r}^{2} \Gamma \sigma_{s r, i}^{-2} \sigma_{r d, i}^{2}+\sigma_{n, d}^{2}}+\left(\mathbf{Q}^{-1}(i, i)\right)^{-1}\right)^{-1}} .
$$

\section{Simulation results}

In this section, we discuss the simulations conducted to estimate the performance of the proposed scheme for an NAF MIMO relaying system. To achieve perfect synchronization, we assume that all CSI's links are known at all nodes with Rayleigh flat fading distribution. Here, we use a quaternary phase-shift keying modulation scheme. Furthermore, we consider a symmetric MIMO relay system, i.e., $N_{S}=N_{R}=N_{D}=4$.

Firstly, we compare the simulation results for AF and NAF MIMO relaying systems. Figure 2 shows a comparison of BER for the AF-based MMSE precoded scheme in [18] and the NAF MIMO relaying systems with and without precoded MMSE. As can be seen, our proposed scheme, i.e., the NAF-based MMSE precoded, outperforms both the AF precoded and NAF unprecoded schemes. It was expected because our design for NAF MIMO relaying scheme is determined by optimizing the transmit power from $S$ and $R$, i.e., $p_{s, i}$ and $p_{r, i}$. Besides that, both source and relay nodes are permitted to transmit their precoded signal simultaneously in both the cooperative and broadcasting phases. In contrast, with the AF MIMO relaying system [18], only relay nodes transmit their precoded signal in the cooperative phase.

Here, we compare BER versus the average SNR performance of the existing unprecoded/precoded scheme for NAF MIMO relaying system. Figure 3 shows the BER comparison for (1) an NAF MIMO relaying system with ZF unprecoded scheme, (2) an NAF MIMO relaying system with precoded based on the ZF criterion [14], (3) an unoptimal NAF MIMO relaying system-based MMSE unprecoded scheme, and (4) the proposed precoded system, i.e., NAF MIMO relaying system based on the MMSE criterion. As can be seen, our proposed precoded scheme outperforms the ZF unprecoded scheme significantly and the previous proposed precoded system [14] slightly. Note that the implementation of ZF precoder scheme in Reference [14] only considered for two-hop AF MIMO system. For a fair comparison, we incorporate the direct link at the destination when performing the MMSE criterion in the simulation process. The superiority of the proposed scheme compared to the unprecoded system is due to the additional source and relay precoder design with the power allocation method, which improves the performance of the NAF MIMO relaying system. 


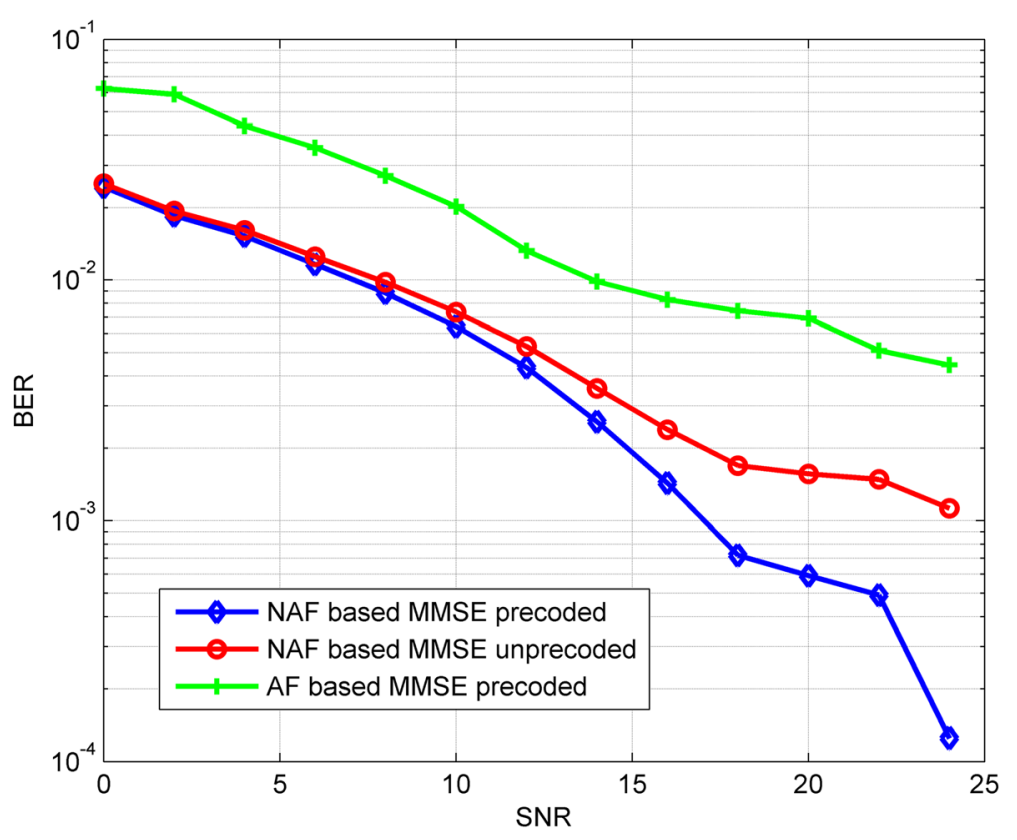

Fig. 2 The performance comparison of NAF with MMSE unprecoded/precoded and AF precoded MMSE in MIMO relaying systems

Finally, we combine the curves in previous simulation results for the comparison of the unprecoded/precoded NAF and AF MIMO relaying system performances. Figure 4 shows the BER comparison for (1) an NAF unprecoded scheme with the ZF criterion, (2) an NAF precoded scheme based on the ZF criterion [14], (3) an NAF precoded scheme based on the MMSE criterion, (4) an NAF unprecoded scheme based on the MMSE criterion, and (5) an AF precoded scheme based on the MMSE criterion [18]. From this figure, we can see that our proposed precoded scheme, i.e., an NAF precoded system based on the MMSE criterion, outperforms not only the unprecoded scheme but also the ZF precoded system in [14]. It is because our proposed scheme incorporates an optimal MMSE precoder design that performance can be enhanced even if the direct link is not

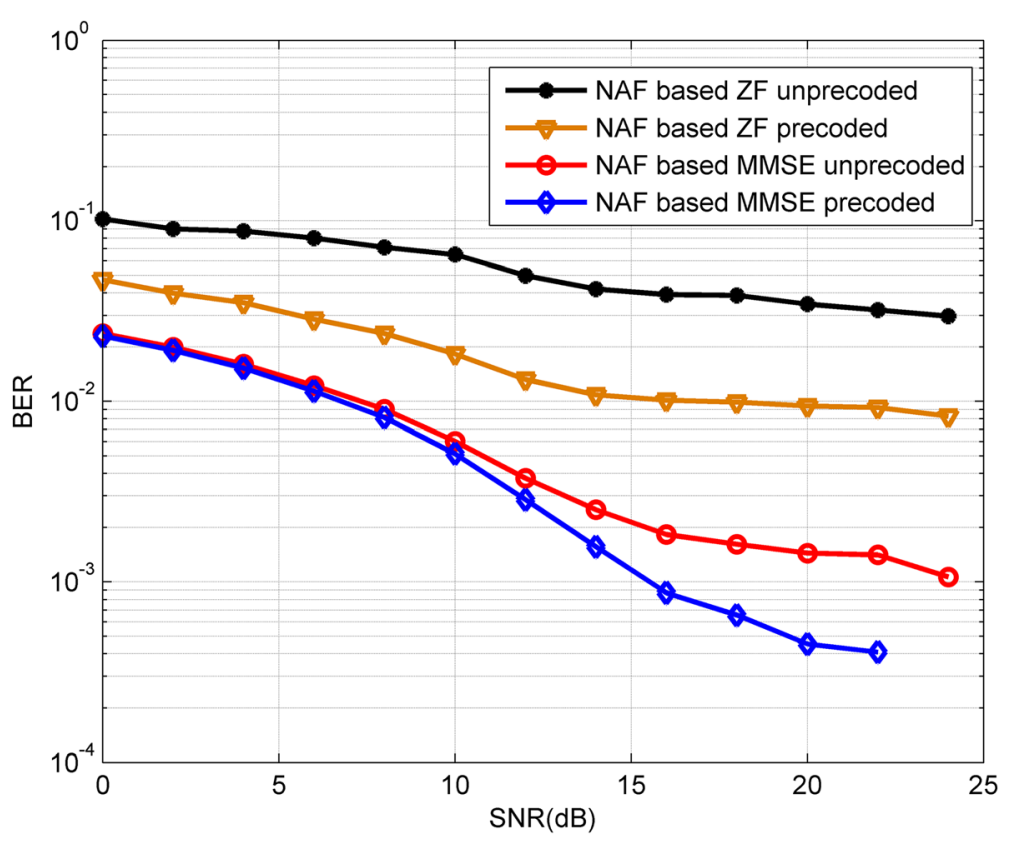

Fig. 3 The performance comparison of NAF MIMO relaying system with MMSE and ZF unprecoded/precoded 


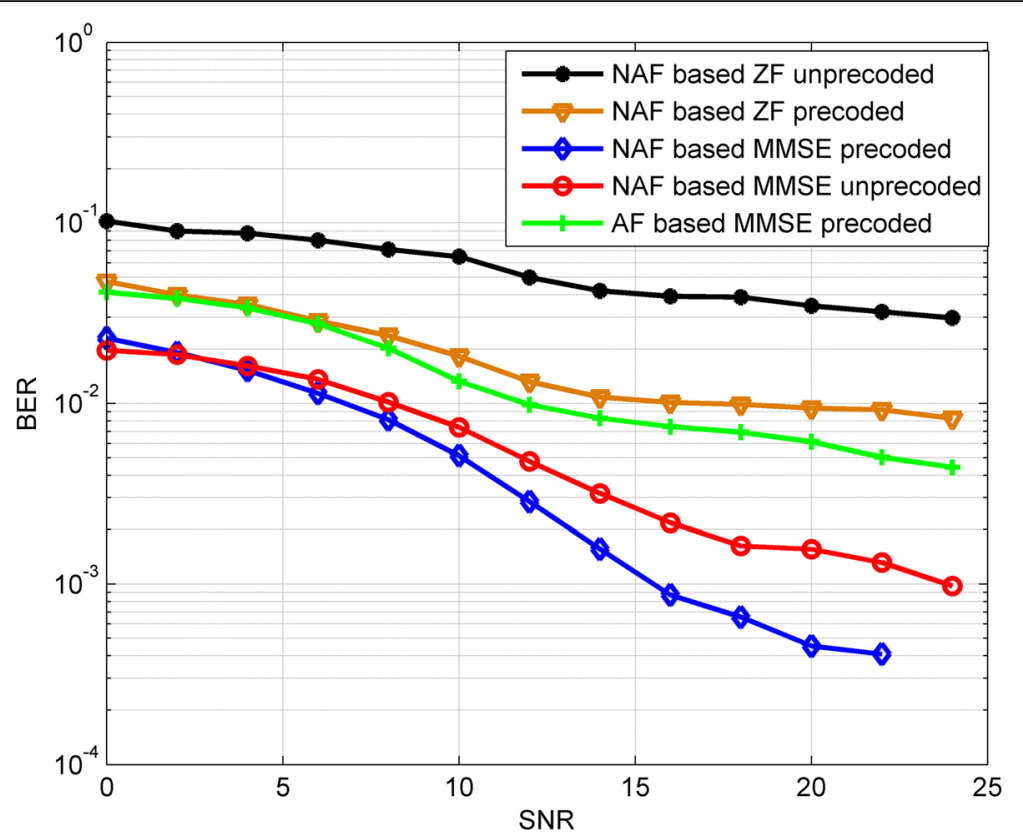

Fig. 4 The performance comparison of the existing unprecoded/precoded in an NAF and AF MIMO relaying system

considered. Besides that, the precoded scheme for AF-based MMSE criterion [18] outperforms both NAF-based ZF precoded [14] and unprecoded schemes. It is due to the MMSE criterion used, in this case, to minimize the total power transmit both from the source and the relay node. So, the performance of the precoded systems with MMSE criterion will be the best among all unprecoded and ZF precoded systems with the same total power transmit.

\section{Conclusions}

In this work, we have proposed a joint design for a linear precoder scheme in a half-duplex non-orthogonal AF MIMO relaying system based on the MMSE criterion. Since MMSE is a detector scheme with high complexity, we obtain an MMSE upper bound using a convenient expression to determine the construction of the precoding matrices using the SVD technique. Simulation results show that the proposed precoded scheme outperforms the unprecoded scheme. By allocating an additional proper power at the source and relay nodes, the proposed scheme increases the performance of the NAF MIMO relaying system compared to $\mathrm{ZF}$ and $\mathrm{AF}$ precoded schemes.

\section{Abbreviations}

AF: Amplify-and-forward; BER: Bit error rate; CSI: Channel state information; DF: Decode-and-forward; MBER: Minimum bit error rate; MIMO: Multiple-input multiple-output; MIR: Maximum information rate; MMSE: Minimum mean square error; NAF: Non-orthogonal amplify-and-forward; OJSRP: Optimal joint source and relay precoding; SNR: Signal-to-noise ratio; SROP: Suboptimal relay only precoding; SVD: Singular-value decomposition; ZF: Zero-forcing

\section{Funding}

This work was supported by a scholarship from the "Magister Program of Education Leading to Doctoral for Excellent Graduates," Ministry of Research, Technology and Higher Education of the Republic of Indonesia, with grant agreement code 67/UN11.2/PP/SP3/2018.

\section{Availability of data and materials}

We decided that the data does not need to be shared since all data that has been obtained through the simulation result using the MATLAB program has been represented in Figs. 2, 3, and 4.

Authors' contributions

YY conceived and designed the study. YY performed the experiments and wrote the paper. NN, MR, and AY reviewed and revised the manuscript. All authors read and approved the manuscript.

\section{Competing interests}

The authors declare that they have no competing interests.

\section{Publisher's Note}

Springer Nature remains neutral with regard to jurisdictional claims in published maps and institutional affiliations.

Received: 5 December 2017 Accepted: 12 November 2018 Published online: 10 December 2018

\section{References}

1. A. Sendonaris, E. Erkip, B. Aazhang, User cooperation diversity part I: system description. IEEE Trans. Commun. 51, 1927-1938 (2003)

2. A. Sendonaris, E. Erkip, B. Aazhang, User cooperation diversity part II: implementation aspects and performance analysis. IEEE Trans. Commun. 51, 1939-1948 (2003)

3. T. Wang, A. Cano, G.B. Glannakis, J.N. Laneman, High-performance cooperative demodulation with decode-and-forward relays. IEEE Trans. Commun. 6, 1427-1438 (2007)

4. J. Luo, R.S. Blum, L.J. Greenstein, A.M. Haimovich, Decode-and-forward cooperative diversity with power allocation in wireless networks. IEEE Trans. Wirel. Commun. 6, 793-799 (2007)

5. G. Kramer, M. Gastpar, P. Gupta, Cooperative strategies and capacity theorems for relay networks. IEEE Trans. Inf. Theory 51, 3037-3063 (2005) 
6. S. Sherstha, K. Chang, Analysis of outage capacity performance for cooperative DF and AF relaying in dissimilar Rayleigh fading channels. IEEE Int. Symp. Inform. Theory, 494-498 (2008)

7. Y. Ding, J. Zhang, K.M. Wong, Ergodic channel capacities for amplify-andforward half-duplex cooperative systems. IEEE Trans. Inf. Theory 55, 713-730 (2009)

8. R.U. Nabar, H. Bölcskei, F.W. Kneubuhler, Fading relay channel: performance limits and space-time signal design. IEEE J Selected Areas Commun. 22, 1099-1109 (2004)

9. B. Gedik, M. Uysal, Impact of imperfect channel estimation on the performance of amplify-and-forward relaying. IEEE Trans. Wirel. Commun. 5 , 1468-1478 (2009)

10. B. Wang, J. Zang, A.H. Madsen, On the capacity of MIMO relay channels. IEEE Trans. Inf. Theory 51, 29-43 (2005)

11. H. Bölcskei, R. Nabar, O. Oyman, A.J. Paulraj, Capacity scaling laws in MIMO relay networks. IEEE Trans. Wirel. Commun. 5, 1433-1444 (2006)

12. R. Mo, Y.H. Chew, MSSE-based joint source and relay precoding design for amplify-and-forward MIMO relay networks. IEEE Trans. Wirel. Commun. 8 , 4668-4676 (2009)

13. A. Danaee, H.R. Bahrami, M. Sadeghzadeh, Precoder design and node power allocation in multi-antenna amplify-and-forward (AF) relay systems. IEEE Int. Conf. on Commun., 5527-5531 (2013)

14. C. Song, C. Ling, On the diversity of linear transceivers in MIMO AF relaying systems. IEEE Trans. Inf. Theory 62, 272-289 (2016)

15. Q.Z. Ahmed, K.H. Park, M.S. Alouini, S. Aissa, Optimal linear detector for nonorthogonal amplify-and-forward protocol. IEEE Int. Conf. Commun., 4829-4833 (2016)

16. Q.Z. Ahmed, K.H. Park, M.S. Alouini, S. Aissa, Linear transceiver design for nonorthogonal amplify-and-forward protocol using a bit error rate criterion. IEEE Trans, Wireless Commun 13, 1536-1276 (2014)

17. D.S. Bernstein, Matrix mathematics (Princeton Univ. Press, Princeton, NJ, 2005)

18. F.S. Tseng, W.R. Wu, Linear MMSE transceiver design in amplify-and-forward MIMO relay systems. IEEE Trans. Vech. Tech. 59, 754-765 (2010)

\section{Submit your manuscript to a SpringerOpen ${ }^{\circ}$ journal and benefit from:}

- Convenient online submission

- Rigorous peer review

- Open access: articles freely available online

High visibility within the field

- Retaining the copyright to your article

Submit your next manuscript at $\boldsymbol{\nabla}$ springeropen.com 